The Third Asia-Pacific Conference on Combustion

June 24-27, 2001, Seoul, Korea

\title{
Combustion in Microscale Heat-Recirculating Burners
}

\author{
Lars Sitzki, Kevin Borer, Ewald Schuster and Paul D. Ronney \\ Dept. Aerospace and Mechanical Engineering \\ University of Southern California, LA, CA 90089-1453 USA
}

\author{
Steffen Wussow \\ Institute of Fire Department, Saxony - Anhalt \\ 39175 Heyrothsberge, Germany
}

\begin{abstract}
In order to optimize the design of microscale combustors, macroscale spiral counterflow heat-recirculating "Swiss Roll" burners were constructed and tested using hydrocarbon fuels at the Reynolds and Damkhler numbers typical of desired microscale values. Both 2D Swiss Roll burners (basically a linearly extruded spiral shape) and fully 3D Swiss Roll burners (in which the spiral is extruded in a circular pattern to create a toroidal geometry) were built using a ceramic "rapid prototyping" technique. It was found that combustion could be sustained in a low-temperature "flameless" mode in which no visible flame occurs. Mixtures well below the conventional lean flammability limit could be burned even at mean flow velocities 30 times the stoichiometric laminar burning velocity. The addition of catalytic materials in the combustion region was found to either increase or decrease the range of flammable mixtures, by substantial amounts in both cases, depending on the Reynolds number. The possibility of using fuels that are selfstarting (i.e. require no external ignition source) on catalytic surfaces was also explored. Preliminary numerical simulations compared rather poorly with the experimental results, most likely due to inaccurate heat loss and chemical reaction rate (both gasphase and surface) sub-models. It is concluded that combustion in microscale burners is feasible, however, heat recirculation, catalysis and careful management of heat losses are essential to the success of such designs.
\end{abstract}

Keywords: combustion, extinction limits, MEMS

\section{INTRODUCTION}

It is well known that the use of combustion processes for electrical power generation provides enormous advantages over batteries in terms of energy storage per unit mass and in terms of power generation per unit volume, even when the conversion efficiency in the combustion process from thermal energy to electrical energy is taken into account. For example, hydrocarbon fuels provide an energy storage density of typically $45 \mathrm{MJ} / \mathrm{kg}$, whereas even modern lithium ion batteries commonly used in laptop computers provide only about $0.50 \mathrm{MJ} / \mathrm{kg}$. Thus, even at only $10 \%$ conversion efficiency from thermal to electrical energy, hydrocarbon fuels provide 10 times higher energy storage density than batteries. For this reason automotive and aviation vehicles employ internal combustion engines for prime moving and electrical power generation almost entirely to the exclusion of batteries, even in vehicles whose mass may be less than $1 \mathrm{~kg}$ or more than $10^{5} \mathrm{~kg}$. Still, these advantages of combustion processes have not yet been exploited for the generation of electrical power in small-scale systems such as laptop computers, cell phones and Micro Electro-Mechanical System (MEMS) devices. The ultimate goal of our current efforts is to develop combustion-driven power generation devices at MEMS-like scales.

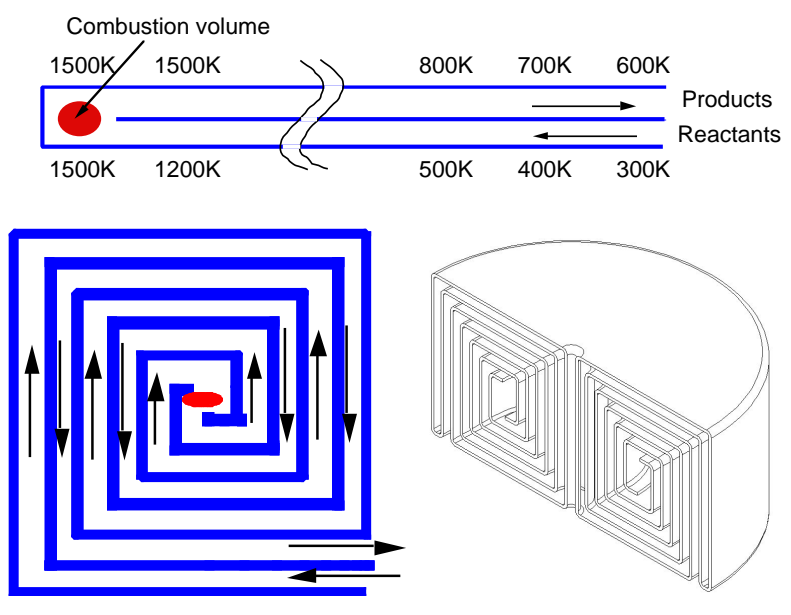

Figure 1. Schematics of excess enthalpy combustors. Upper: generic linear counterflow heat exchanger / combustor with typical temperatures shown; lower left: linear device rolled up into a "two dimensional" Swiss Roll combustor; lower: cutaway view of "three-dimensional " toroidal Swiss Roll device.

Most current micro-scale combustion generator concepts employ scaled-down versions of existing macroscale devices, in particular internal combustion engines, though such microdevices experience more difficulties with heat losses, friction, sealing, fabrication, assembly etc. than their macroscale counterparts. Because of these limitations, we have focussed our attention on a power source having no moving parts and not requiring highprecision fabrication and assembly. Consequently, we have chosen a heat-recirculating combustor design. Electrical power is generated by thermoelectric elements embedded in walls between cold incoming reactants and hot outgoing products. The complicated geometry can be monolithically fabricated at microscale by using EFAB (Electrochemical FABrication) technology [1]. By this method, one can produce arbitrary 3-D structures by stacking hundreds of individually patterned layers. For brevity, in this paper only the combustion aspects of our project are presented, which may be applicable to other types of microscale heat and/or power generation systems. The combustion approach is tested using macroscale prototypes. 


\section{APPROACH}

Weinberg and collaborators [2] have shown that quenching limits can be effectively eliminated through the use of recirculation of thermal energy from the combustion products to preheat the reactants without recirculating the combustion products themselves. Such devices are termed "excess enthalpy" burners because the thermal enthalpy of the incoming reactants is increased via preheating without diminishing the chemical enthalpy via chemical reaction, thus at the point within the device that combustion does occur, the reactants can have much higher total enthalpy (chemical plus thermal) than the fresh reactants. Figure 1 (upper) shows a generic schematic of an excess enthalpy combustor. The large external surface area of such a heat exchanger would typically lead to large heat losses to ambient, but by wrapping up the heat exchanger in a Swiss roll configuration (Fig. 1, middle) such heat losses are effectively eliminated and in fact the heat transfer between windings becomes an integral part of the burner design. Of course, for such a burner to work, the rate of heat conduction outward across the turns of the burner must be slower than the rate at which heat is convected inward. The ratio of convective to conductive transfer is proportional to $U d / \alpha$, where $U$ is the gas velocity in the channel, $d$ the channel width and $\alpha$ is the thermal diffusivity of the gas. Since for gases $\alpha^{-} v$, where $v$ is the kinematic viscosity, this is equivalent to requiring that the Reynolds number $\mathrm{Re} \equiv \mathrm{Ud} / \mathrm{v}$ be sufficiently larger than unity. Consequently, as long as Re is sufficiently large, combustion can be sustained in Swiss-roll type burners in micro-scale devices even though combustion could not possibly be sustained in devices of channels with cold walls except at very high pressures and/or with walls preheated to impracticably high temperatures. Even in a Swiss roll configuration, a planar roll will suffer large heat losses in the lateral direction. A mechanism to avoid this, which was in fact proposed by Lloyd and Weinberg (1975), is to wrap the Swiss roll in the lateral direction as well to create a toroidal shape (Fig. 1, lower). While this is difficult with virtually all prior MEMS technologies which allow only for the fabrication of quasi-two-dimensional structures, it is possible to build such a three-dimensional microstructure using the EFAB technique to be used in this project.

An additional means to reduce the impact of heat loss in microscale systems is through the use of catalytic combustion. In contrast to gas phase combustion, catalytic combustion occurs at a surface reaction without a flame. Catalytic combustion can serve several useful purposes in this context. Since chemical reactions only occur on the catalyst surface, the location of the heat source is fixed. This makes heat transfer design simpler than gas-phase combustion in which the location of reaction zone may change in undesirable ways. Furthermore, in MEMS combustors, the higher surface area to volume ratio makes catalytic combustion even more attractive. Finally, the lower temperature of catalytic combustion makes thermal stresses, materials limitations and heat losses less problematic.

\section{EXPERIMENTAL APPARATUS}

Because it is difficult to measure combustion properties (e.g. temperature, composition) in microscale devices, we have chosen to build first macro- and meso-scale toroidal Swiss-roll burners (without thermoelectric devices) which are more readily instrumented. Both 2D burners (basically a linearly extruded spiral shape) and fully 3D burners (in which the spiral is extruded in a circular pattern to create a toroidal geometry) have been built. For the macroscale devices, the typical channel size of the macroscale test devices is $3 \mathrm{~mm}$ vs. $0.13 \mathrm{~mm}$ for the micro-scale device we propose. The macro-scale devices (Fig. 2) were built using Solidgen" rapid prototyping techniques using sintered alumina-silica with a typical $20 \mathrm{~m}$ pore size (as opposed to the electrochemical process of EFAB). It is readily shown that with this pore size the inter-turn gas leak-through is negligible. For the meso-scale test devices (Fig. 3) of typical channel dimension $0.4 \mathrm{~mm}$ were built using wire electrodischarge machining (EDM). Considerable effort was expended to find appropriate materials and manufacturing techniques. Since the wire EDM process can only create two-dimensional extrusion-like structures, end plates are attached for 2D versions, and 6 pie-shaped pieces are butted together to form a quasitoroidal 3D part. For the meso-scale burners, the combustion chamber region is smaller than $1 \mathrm{~mm}$ and is therefore smaller than the quenching distance for stoichiometric mixtures. A manifold for the fresh mixture is attached to the combustors and a frame is used to hold the various parts of the combustor and manifold together.
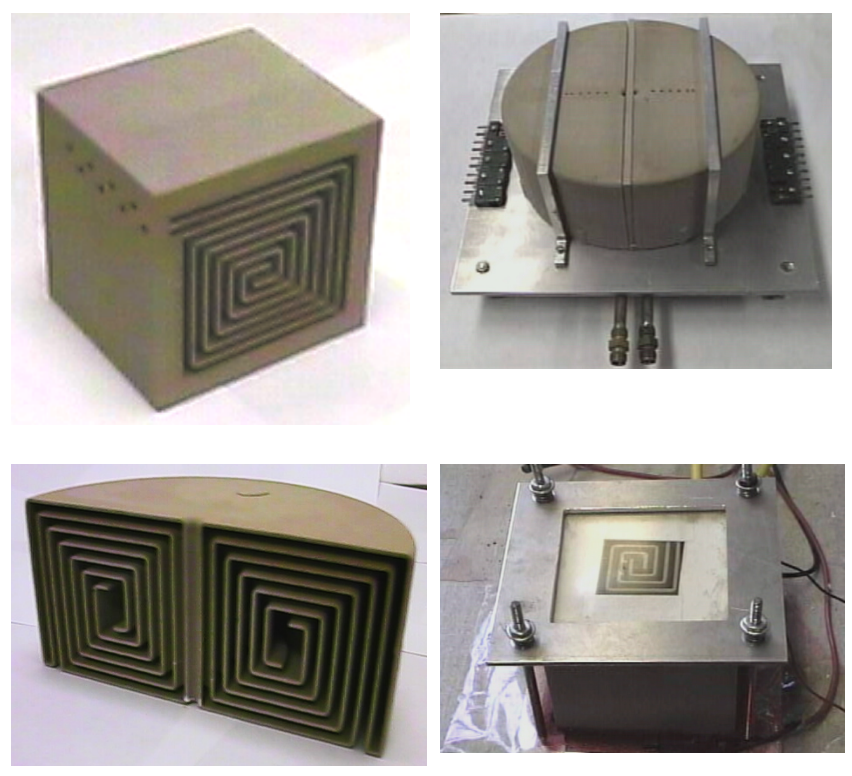

Figure 2: Images of macroscale burners. Upper left: $2 D$ Swiss roll part built using rapid prototyping; lower left: $3 D$ Swiss roll half- torus built using rapid prototyping; upper right: $3 D$ burner (two halves) in experimental configuration; lower right: $2 D$ burner in experimental configuration.

A PC with LabView software is used to control mass flow controllers for fuel and air and to record data from thermocouples for temperature measurement. The exhaust gas composition was analyzed with a gas chromatograph. Electrically heated Kantal wire is used for ignition.

\section{EXPERIMENTAL RESULTS}

Figure 4 shows the minimum propane concentration supporting self-sustaining combustion as a function of the areaaveraged flow velocity in the channels. Data for butane and dimethyl ether (not shown) exhibited similar trends. It can be 
seen in Fig. 4 that mixtures well below the conventional lean flammability limit can be burned even at mean flow velocities 10 times the stoichiometric laminar burning velocity $(40 \mathrm{~cm} / \mathrm{s})$. The high velocity extinction limit is almost certainly due to insufficient residence time compared to the chemical reaction time scale (analogous to blowing out a candle flame). These data are replotted as the fuel enthalpy flux (which is proportional to flow velocity multiplied by the fuel concentration) as a function of the Reynolds number based on channel width (which is proportional to flow velocity) in Fig. 5. It can be seen that the lower velocity limit corresponds to a constant enthalpy flux, and thus a constant ratio of heat loss to heat generation, which is indicative of a limit due to heat loss. Figure 5 shows that Reynolds number as least as low as 20 can support combustion with heat recirculation and catalytic combustion (discussed later).
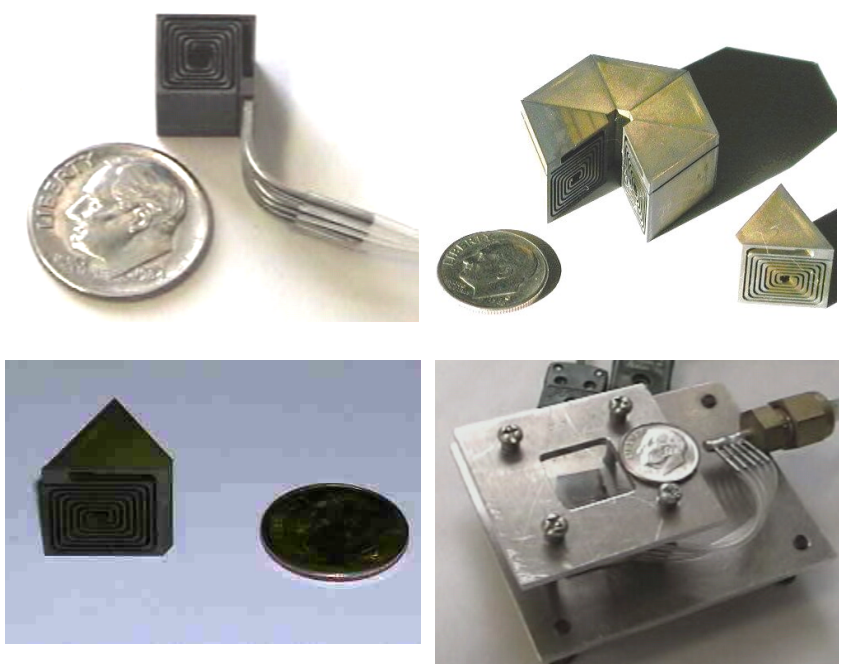

Figure 3: Images of mesoscale burners. Upper left: $2 D$ Swiss roll part built using wire EDM; lower left: $3 D$ wedge section; upper right: $3 D$ burner (six wedge-shaped parts); lower right: $2 D$ burner in experimental configuration.

Interestingly, it was found that under most conditions, even without catalysts, combustion occurs in a "flameless" mode in which no visible flame occurs. Recently other investigators [3] have observed similar behavior in macroscale combustors using highly preheated air, though the reason for the differences between flameless and conventional burning have not yet been established. Perhaps even more surprising is that the addition of platinum strips as a catalytic material in the combustion region can either increase or decrease the range of flammable mixtures, by substantial amounts in both cases, depending on the flow velocity. This is likely due to the catalyst acting as either a net source of radicals at low velocities where the residence times are large and temperatures are lower, or a net sink of radicals at higher flow velocities where the residence times are shorter and temperatures higher. Figure 4 shows that nickel and palladium strips and copper strips plated with platinum have catalytic activity, but less than platinum strips.

Figure 6 shows that the minimum temperature supporting combustion (in the center of the combustor) is much lower when catalysts are used, less than 200ßC in some cases. Note that even without catalyts, the temperature required to support combustion (typically 800ßC) is lower than that required to support propagating flames (typically 1200ßC).

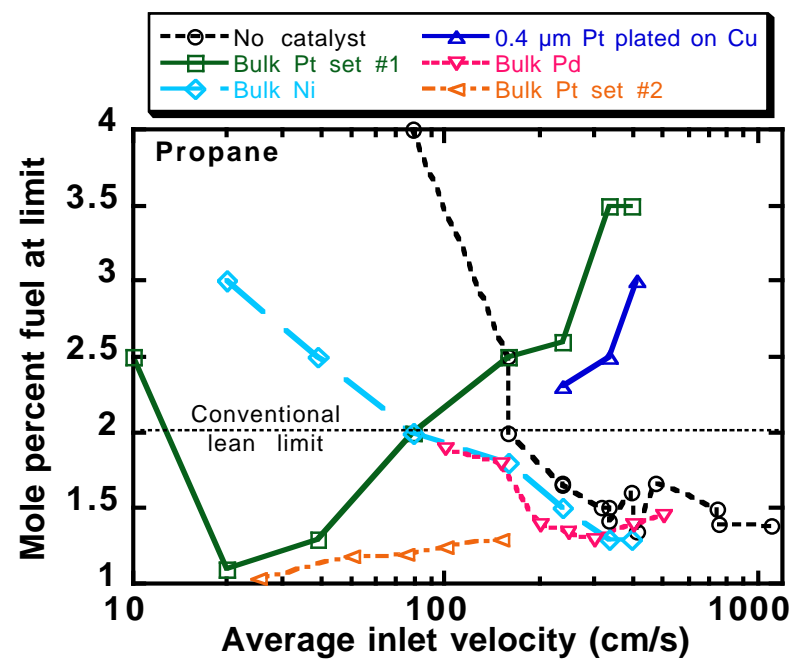

Figure 4. Effect of area-averaged flow velocity in the 2-D mesoscale burner on the minimum fuel concentration for selfsustaining combustion.

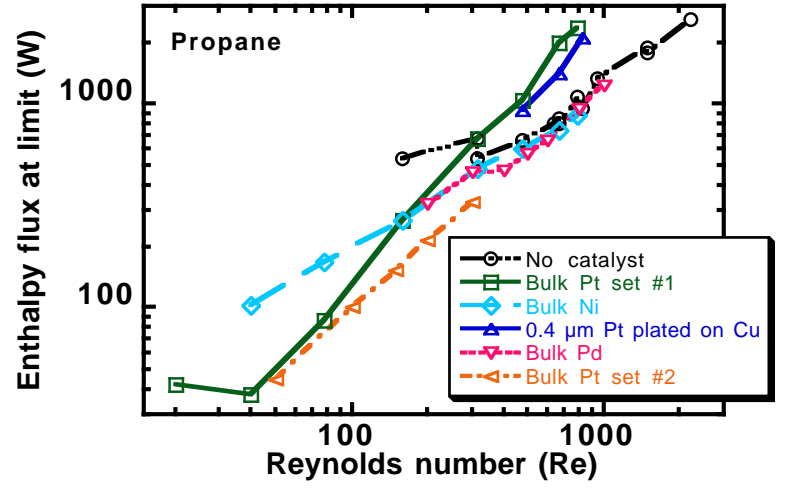

Figure 5. Same data as Fig. 4, plotted in terms of the input chemical enthalpy flux as a function of the Reynolds number based on channel width.

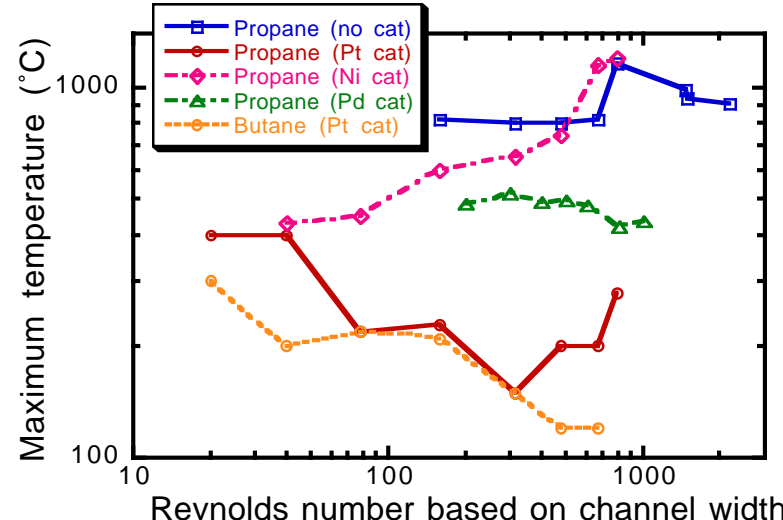

Figure 6. Maximum gas temperature in the macroscale burner as a function of the Reynolds number based on channel width.

Different fuels were examined to determine if any are "selfstarting" in the presence of catalysts, i.e. will ignite without preheating the combustor. Self-starting fuels and catalysts are highly desirable because it would eliminate the need for glow plugs, supplemental battery, electronics, etc. associated with active ignition systems, or at least reduce the ignition power 
requirements. Even if self-starting at room temperature is not achieved, ignition temperatures well below maximum allowable temperatures for thermoelectric devices have been obtained. Consequently, a set of tests was performed in which the entire burner was heated electrically to a temperature above the desired test temperature with air only flowing through the burner, then the electrical power was removed and when the temperature dropped to the target value, the fuel flow was started. The ignition temperature was defined as the minimum temperature for which a steady reaction evolved after the fuel flow started. Hydrogen fuel was found to be self-starting even at room temperature. Figure 7 shows that dimethyl ether has an ignition temperature as low as $120 ß \mathrm{C}$, as compared to $200 B \mathrm{C}-300 B \mathrm{C}$ for butane and propane. Methane (not shown) was found to have a much higher starting temperature.

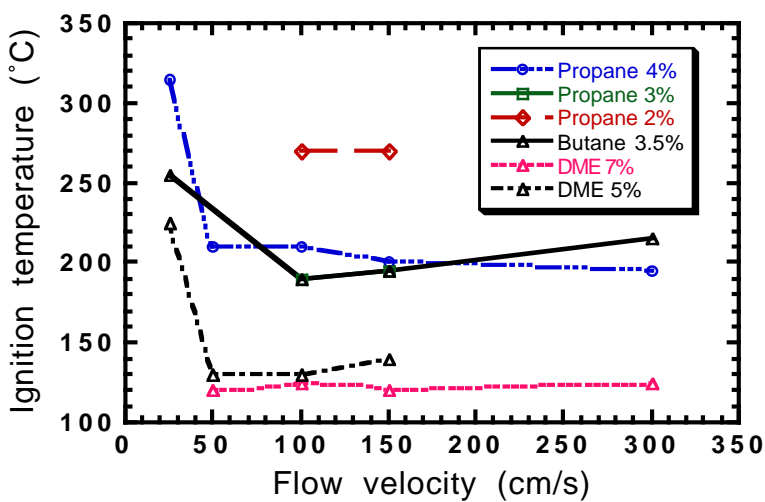

Figure 7. Minimum self-staring temperature in the $2 D$ Swiss toll burner for various fuels and mixture ratios as a function of flow velocity.

Figure 8 shows the temperature in the center of the 2D mesoscale combustor as a function of mixture strength and Reynolds number (thus flow velocity). In the mesoscale combustor, a loop of $\mathrm{Pt}$ wire was used in all tests as a catalyst. Note that at in addition to a fall-off in temperature and eventually extinction at too high or too low fuel concentrations, below $\mathrm{Re}^{-}$ 200 , there is a marked decrease in temperature even for nearstoichiometric $(4.02 \%)$ mixtures. Such behavior is not found for the macroscale burners at this high a Reynolds number. This is likely due to the greater heat loss rate compared to heat generation rate for the mesoscale burner, indicating the greater difficulty of sustaining combustion at microscales, even with heat recirculation, because of the out-of-plane heat losses. This also shows the advantage of the toroidal geometry for microscale combustion because of the lower heat losses.

\section{CONCLUSIONS}

Our preliminary experiments indicate that combustion in microscale combustion devices at low Reynolds numbers is possible, but require heat recirculation via the "Swiss Roll" or similar heat exchanger geometries, as well as catalytic combustion. A flameless mode of combustion was prevalent even without catalysts. With catalysts, the extinction limits narrow at higher Reynolds numbers but broaden substantially at lower $\mathrm{Re}$, which are the conditions most relevant to microscale combustion. Catalytic combustion also leads to much lower selfstarting temperatures. The development and calibration of a gasphase and surface chemistry model is one of the biggest challenges ahead. Preliminary numerical predictions (not shown) of the extinction boundary using a 2D computational fluid dynamics code compare reasonably well with experiments at higher Reynolds numbers but rather poorly at the lower Re. This is most likely due to the fact that the out-of-plane heat losses, which account for most of the losses, are not accounted for in the 2D model. This will be remedied by using a full 3D model. Additional challenges are controlling of the final device and auxiliary components like valves, pumps and fuel tanks.

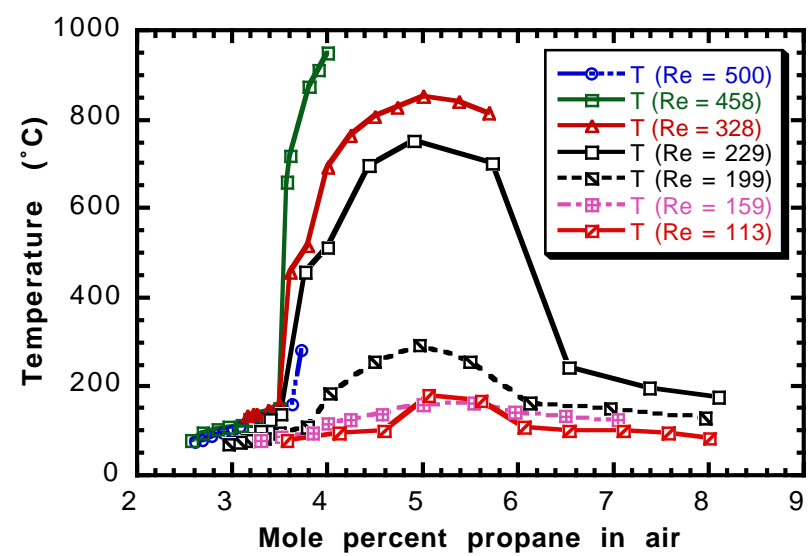

Figure 8. Maximum gas temperature in the mesoscale burner using propane fuel as a function of the Reynolds number based on channel width.

\section{ACKNOWLEDGEMENTS}

This work was supported by the U. S. Defense Advanced Research Projects Agency (DARPA) Microsystems Technology Office under Contract \#DABT63-99-C-0042 to USC.

\section{REFERENCES}

1. Cohen, A.: EFAB: Rapid, Low-Cost Desktop Micromachining of High Aspect Ratio True 3-D MEMS, 12th IEEE International Microelectromechanical Systems Conference, 1999; Cohen, A.: 3-D Micromachining by Electrochemical Fabrication, Micromachine Devices, March 1999.

2. Jones, A. R., Lloyd, S. A., Weinberg, F. J. (1978). Combustion in heat exchangers, Proc. Roy. Soc. (London) A 360, 97-115; Lloyd, S. A. and Weinberg, F. J. (1974). A burner for mixtures of very low heat content, Nature 251, 47-49; Lloyd, S. A. and Weinberg, F. J. (1975). Limits to energy release and utilisation from chemical fuels, Nature 257, 367370.

3. Wnning, J. A., Wnning, J. G., " Flameless oxidation to reduce thermal NO-formation," Prog. Energy Combust. Sci. 23, 81-94 (1997); Katsui, M., Hasegawa, T., "The science and technology of combustion in highly preheated air," Proc. Combust. Inst. 27, 3135-3146 (1998); Maruta, K., Muso, K., Takeda, K., Niioka, T., "Reaction zone structure in flameless combustion," Proc. Combust. Inst. 28, in press (2000). 